\title{
Neues Instrument der Umweltpolitik
}

\section{Das Fluss(einzugs)gebietsmanagement lässt sich dem neuen Instrument des Ökosystemmanagements zuordnen. Ähnlich wie anderen neven Ansätzen wer- den kooperative Lösungen lokaler und regionaler Akteure in den Vordergrund gestellt. Die Erfolgsaussichten eines solchen Vorgehens werden von neven the- oretischen Konzepten untermauert, sofern auf der darüber liegenden Ebene eine Begleitung und notfalls auch Sanktionierung erfolgt.}

D Von Ulrich Petschow er überkommene command and controlAnsatz der Umweltpolitik stößt an Grenzen: Mit diesem gelingt es nicht, die verschiedenen komplex miteinander verbundenen Probleme in stärker systematischer und vorausschauender Weise anzugehen. Beispielsweise versucht dieser Ansatz komplexe Probleme durch Fragmentierung zu lösen, mit der Folge vielfach unerwünschter Nebenwirkungen und ineffizienter Maßnahmen. Die Kritik an den Ergebnissen dieser Form der Umweltpolitik sowie das Aufkommen der Idee der nachhaltigen Entwicklung fuihrte zu einer Art institutioneller Antithese der bisherigen Regulierung. So wird darauf verwiesen, dass sich eine neue Phase der Umweltpolitik entwickelt. Deren Erfolge sind allerdings bislang nur schemenhaft zu bewerten, vor allem da sie sich meist auf erfolgreiche Fallbeispiele beziehen und damit nur begrenzt verallgemeinerbar sind. Diese neuen Ansätze fokussieren unter anderem auf folgende Instrumente:

1. Die Integration von Umweltaspekten in andere Politikbereiche,

2. Anforderungen an die Veröffentlichung von Umweltdaten,

3. Umweltmanagementsysteme,

4. Ökosystemmanagement,

5. Zertifizierungen,

6. Verhandlungssysteme,

7. codes of conduct (Verhaltenskodizes) und

8. freiwillige Vereinbarungen.

Zumindest ein Teil der benannten neuen Instrumente versucht, verstärkt die Problemlösungsfähigkeiten der nicht-staatlichen Akteure - unter Einbeziehung der Problemverursacher - zu nutzen und ist in der Regel weniger auf direkte Regulierungen orientiert. Geht man davon aus, dass Umweltprobleme letztlich Probleme der sozialen Organisation sind (Norgaard), ist dieser Schritt folgerichtig.
Das Flusseinzugsgebietsmanagement lässt sich dem Ökosystemmanagement zuordnen, indem versucht wird das nachhaltige Management der Wasserressourcen in einem Naturraum zu erreichen. Das Ökosystemmanagement wird teilweise mit dem Ansatz des Bioregionalismus in Verbindung gebracht, in welchem der Naturraum eine wesentliche Klammer des regionalen Kontextes und damit handlungsorientierend sein könnte. In diesem Sinne wäre von einer Rückbindung beispielsweise der wirtschaftlichen Entwicklung an die naturräumlichen Bedingungen zu sprechen. Das Flusseinzugsgebietsmanagement beinhaltet zumindest Elemente dieses Ansatzes.

Wichtige Elemente, die in diesem Kontext für eine Veränderung der Governancemechanismen sprechen, sind

1. die Probleme des Umgangs mit diffusen Emissionsquellen, die sich dem herkömmlichen Ansatz zumindest zum Teil entziehen,

2. die Betrachtung von Naturräumen als komplexe Systeme, die systemar, im Sinne eines integrierten ökosystemspezifischen (und damit vielfach regionalen) Ansatzes adaptiv und damit flexibel gemanagt werden müssen (1), und damit

3. auf das Wissen vor Ort fokussieren und die damit verbundenen potenziellen Problemlösungsfähigkeiten sowie

\section{Digital(er Struktur- wandel $)=$ nachbaltig $($ er Strukturwandel)?}

ist das Thema des Informationsdienstes

\section{Ökologisches Wirtschaften 3-4/02}

Wenn Sie potenzielle Beiträge haben, wenden Sie sich bitte an die Redaktion.
4. die Chance eröffnen, durch alternative Governanceinstitutionen letztlich auch die Transaktionskosten reduzieren zu können.

\section{Neve Kooperationsstrukturen ent- wickeln}

Folglich erweist sich die Integration der vielfältigen Wechselwirkungen von umweltorientierten, ökonomischen und sozialen Interessen als zentral, damit die wesentlichen Akteure ihre Entscheidungen vor dem Hintergrund eines gemeinsamen ökologischen Kontextes treffen. Dies erfordert bottom up-Prozesse sowie eine umfassende Einbeziehung der Stakeholder. Ein entscheidendes Element ist dabei, in einem komplexen System wie Flusseinzugsgebieten ein gemeinsames Verständnis von den Problemen bzw. auch von den Ursache-Wirkungs-Beziehungen zu entwickeln - und damit die kognitiven Strukturen zu verändern. Vor diesem Hintergrund kommt nicht zuletzt auch der Wissenschaft die wichtige Rolle zu, Unsicherheiten zu mindern und damit die Legitimität von Problemlösungsansätzen zu erhöhen. Entsprechende Studien aber auch Modellierungen haben in solchen Managementkonzepten eine wesentliche Funktion zur Verständigung aber auch zur möglichen Maßnahmeentwicklung (vgl. auch den Beitrag von Welp in diesem Heft)

Damit ist es ein zentrales Problem dieser Ansätze, geeignete Kooperationsstrukturen und Regelungsmechanismen zur Lösung von Umweltproblemen im Flusseinzugsgebiet zu entwickeln. Diese Frage hat unter dem Stichwort Überwindung der Dilemmata der Übernutzung von common pool resources (CPR) eine lange $\mathrm{Ge}$ schichte. Die Hardinsche tragedy of the commons erweist sich bei genauerer Betrachtung als Problem des unbeschränkten Zugangs (2). Ostrom hat anhand einer Vielzahl von Beispielen aufgezeigt, dass in vielen Bereichen von den lokalen Akteuren Regelungsmechanismen zum Management der CPR entwickelt werden konnten, die eine nachhaltige Bewirtschaftung der Ressourcen ermöglicht haben (3). Staatliche Vorgaben haben in einer Vielzahl von Fällen hingegen geradezu kontraproduktive Effekte erzeugt, indem es zu einem ,,crowding out“ von regionalen Governancemechanismen gekommen ist.

Flusseinzugsgebiete können als CPR begriffen werden. Diese unterliegen im Grundsatz dem Problem der Übernutzung - wie es auch gegenwärtig der Fall ist. Damit stellt sich die Frage nach geeigneten 
institutionellen Arrangements, die, wie beschrieben, durchaus nicht allein auf zentralstaatlicher Ebene gefunden werden müssen bzw. gar können. Unter der Annahme rationaler, das heißt auf Eigeninteresse orientierter Akteure erscheinen kooperative Lösungen kaum denkbar, dementsprechend wird die Lösungskompetenz entsprechender Ansätze vielfach in Frage gestellt. Die experimentelle Ökonomie zeigt hingegen auf, dass bereits die Zulassung der Möglichkeit der Kommunikation als auch von informellen Sanktionen, aber auch die Einbeziehung von Reziprozitätsannahmen, die Wahrscheinlichkeit der Kooperation deutlich erhöht (4). Bezieht man in diesen Kontext auch evolutionäre Ansätze mit ein, beispielsweise Konzepte wiederholter Spiele mit den gleichen Akteuren, so sind mit höherer Wahrscheinlichkeit kooperative Ansätze mit entsprechend veränderten kognitiven Strukturen die Folge.

\section{Veränderte \\ Governancemechanismen}

Dies bedeutet in Bezug auf die oben angegebenen Ansätze, dass zum Beispiel das Flusseinzugsgebietsmanagement vor dem Hintergrund der Institutionen-Entwicklung auf dieser Ebene - also der Integration von Politikbereichen wie auch der Einbeziehung der Stakeholder - die Basis für einen kontinuierlichen Austausch, die Diskussion und auch eine (Selbst-) Verpflichtung darstellen kann. Es hängt allerdings entscheidend von der Form der Institutionalisierung und der Partizipationsansätze $a b$, inwieweit damit tatsächlich weiter reichende Lösungen ausgelotet und erreicht werden können.

Damit werden veränderte, ,hybride“ Governancemechanismen erforderlich, die von der Vorstellung des umfassend regulierenden Staates Abschied nehmen müssen. Karkkainen betont in diesem Kontext, dass eine Neudefinition der Kernattribute der staatlichen Souveränität nicht alleine auf die internationale Ebene beschränkt ist, sondern dass vielmehr generell ein anderes, problemlösungsorientiertes ,polyarchic governance model" erforderlich ist (5). Ein Modell, das auf lokal oder regional zugeschnittenen integrativen, kooperativen und zugleich experimentellen Arrangements basieren müsste.

Fung und Wright verbinden diese neuen Ansätze - nicht allein auf ökologische Fragen bezogen - mit dem veränderten Demokratieverständnis einer so genannten empowered deliberative democracy (6). Ausgehend von der beschriebenen Problemlage und den kom- plexer werdenden Bedingungen muss der zunehmenden Heterogenität Rechnung getragen werden, indem

1. auf Kommunikation und öffentliche Rechtfertigung fokussiert wird,

2. die Erfahrungen des zivilgesellschaftlichen Engagements einbezogen werden und schließlich 3. auf die Diskussionsstränge zu neuen Governanceformen Bezug genommen wird.

\section{- Regionalismus unter Supervision}

Damit werden Problemorientierung und partizipative bottom up-Prozesse betont. Fung und Wright heben dabei einen wesentlichen Unterschied ihres Ansatzes gegenüber den herkömmlichen Vorstellungen der Verlagerung der Verantwortlichkeiten auf „untere“ Ebenen hervor: Entscheidend sei, dass diese Verlagerung durch Prozesse der Supervision und Koordinierung begleitet werde. Sie bleibt damit in staatlicher Verantwortung und sollte nicht im Sinne einer umfassenden Deregulierung, Privatisierung und Freiwilligkeit verstanden werden.

Hier würden bezogen auf unser Beispiel die Aufgaben aber auch die Maßnahmen des Flusseinzugsgebietsmanagements auf regionale Akteure verlagert. Diese können eigene Maßnahmen und Instrumente nutzen, müssen aber zugleich generelle Zielvorgaben erreichen. Damit werden Lernprozesse auf lokaler und regionaler Ebene angeregt, deren wesentliche Effekte vor allem durch Benchmarking-Prozesse und den Vergleich mit anderen Regionen erreicht werden können. Hier kommt insbesondere dem Monitoring und den darauf basierenden Lernprozessen eine wesentliche Aufgabe zu, indem die Umsetzungsprozesse und deren Erfolg bzw. Misserfolg kontinuierlich überprüft werden und damit zu einer Anpassung der Maßnahmen führen.

\section{WRRL im Ansatz viel versprechend}

Zumindest in Teilbereichen entspricht die Vorgehensweise der WRRL diesen Prinzipien. Entscheidend ist allerdings, wie diese Ansätze in die Praxis überführt werden, denn die konkrete Vorgaben etwa zur Ausgestaltung der Partizipation sind in der WRRL wenig entwickelt. Innovative Anstöße müssen dem entsprechend gerade auch ,,von unten" angestoßen werden. Inwieweit die Chancen neuer umweltpolitischer Ansätze tatsächlich auch genutzt werden können, hängt daher entscheidend von den konkreten Veränderungen bzw. auch der Veränderungsbereitschaft im Hinblick auf diese Prozesse ab.
Generell wird die Reichweite der neuen umweltpolitischen Instrumente der Partizipation aber auch der Selbstregulierung jedoch noch wenig verstanden. Die Bedingungen erfolgreicher bzw. weniger erfolgreicher Ansätze sind noch unklar. Insofern besteht trotz der Vielzahl von Untersuchungen etwa zu den Agenda 21-Prozessen nicht nur Forschungs-, sondern vor allem auch Experimentierbedarf, um die Realitätstauglichkeit dieser neuen umweltpolitischen Instrumente zu erproben. Vielleicht geht es dabei letztlich vorrangig um mehr oder weniger „Schatten der Hierarchie“ (Scharpf).

Gerade im Zusammenhang mit der Ziel- bzw. Erfolgsorientierung von Verwaltungsreformbemühungen bestünde ein umfassendes Experimentierfeld, dessen Auslotung allerdings offenbar schwer fällt (7).

\section{Anmerkungen}

(1) Holling, C.S./ Berkes, F./ Folke C.: Science, Sustainability and Resource Management. In: Berkes, F./ Folke, C. (Hrsg.): Linking Social and Ecological Systems: Management Practices and Social Mechanisms for Building Resilience. New York 1998. Vgl. auch Holling, C.S./ Gunderson, L.H./ Peterson, G.: Comparing Complex Adaptive Systems. In: Ökologisches Wirtschaften, Nr. 3-4/1998, Spezial S. 4-6.

(2) Fischer, L:: Die Common Property Debatte neu bewertet. Von der "Tragedy" zu den "Benefits" of the Commons. Schriftenreihe des IÖW 104/1996, Berlin.

(3) Ostrom, E.: Die Verfassung der Allmende. Tübingen 1999.

(4) Falk, A./ Fehr, E./ Fischbacher, U.: Appropriating the Commons - a Theoretical Explanation. WP 55, Institute for Empirical Research in Economics, ETH Zürich 2000.

(5) Karkkainen, B.C.: Post Sovereign Environmental Governance: the Collaborative Problem-Solving Model. 2001, mimeo. Zur internationalen Dimension vgl. Petschow, U./ Clausen, J./ Keil, M.: Zivilgesellschaftliche Akteure und Unternehmen im Rahmen von Global Governance. IÖW, Berlin, im Erscheinen.

(6) Fung, A./ Wright, E.0.: Deepening Democracy: Innovations in Empowered Participatory Governance. In: Politics and Society, Vol. 29 (2001), Nr. 1, S. 5-41.

(7) Vgl. hierzu z.B. Donner, S./ Kubala, F./ Petschow, U.: Verwaltungsreform und Nachhaltigkeitsziele. In: Ökologisches Wirtschaften, Nr. 3-4/2000, S. 27-28.

\section{Der Autor}

Ulrich Petschow ist Leiter des Forschungsfelds Umweltökonomie und -politik des Instituts für ökologische Wirtschaftsforschung (IÖW).

Kontakt: IÖW, Potsdamer Str. 105, 10785 Berlin. Tel. 030/884594-0, Fax 030/ 8825439, E-mail: Ulrich.Petschow@ioew.de 
(c) 20I0 Authors; licensee IÖW and oekom verlag. This is an article distributed under the terms of the Creative Commons Attribution Non-Commercial No Derivates License (http://creativecommons.org/licenses/by-nc-nd/3.o/), which permits unrestricted use, distribution, and reproduction in any medium, provided the original work is properly cited. 